\title{
Actinic Keratosis Clinical Practice Guidelines: An Appraisal of Quality
}

\author{
Joslyn S. Kirby, ${ }^{1}$ Thomas Scharnitz, ${ }^{2}$ Elizabeth V. Seiverling, \\ Hadjh Ahrns, ${ }^{3}$ and Sara Ferguson ${ }^{1}$ \\ ${ }^{1}$ Department of Dermatology, Penn State Milton S. Hershey Medical Center, Hershey, PA 17033, USA \\ ${ }^{2}$ Penn State College of Medicine, Hershey, PA 17033, USA \\ ${ }^{3}$ Department of Family and Community Medicine, Penn State Milton S. Hershey Medical Center, Hershey, PA 17033, USA \\ Correspondence should be addressed to Joslyn S. Kirby; jkirbyl@hmc.psu.edu
}

Received 10 July 2015; Revised 20 August 2015; Accepted 31 August 2015

Academic Editor: Jean Kanitakis

Copyright (C) 2015 Joslyn S. Kirby et al. This is an open access article distributed under the Creative Commons Attribution License, which permits unrestricted use, distribution, and reproduction in any medium, provided the original work is properly cited.

\begin{abstract}
Actinic keratosis (AK) is a common precancerous skin lesion and many AK management guidelines exist, but there has been limited investigation into the quality of these documents. The objective of this study was to assess the strengths and weaknesses of guidelines that address AK management. A systematic search for guidelines with recommendations for AK was performed. The Appraisal of Guidelines for Research and Evaluation (AGREE II) was used to appraise the quality of guidelines. Multiple raters independently reviewed each of the guidelines and applied the AGREE II tool and scores were calculated. Overall, 2,307 citations were identified and 7 fulfilled the study criteria. The Cancer Council of Australia/Australian Cancer Network guideline had the highest mean scores and was the only guideline to include a systematic review, include an evidence rating for recommendations, and report conflicts of interest and funding sources. High-quality, effective guidelines are evidence-based with recommendations that are concise and organized, so practical application is facilitated. Features such as concise tables, pictorial diagrams, and explicit links to evidence are helpful. However, the rigor and validity of some guidelines were weak. So, it is important for providers to be aware of the features that contribute to a high-quality, practical document.
\end{abstract}

\section{Introduction}

Actinic keratosis (AK), or solar keratosis, is a common dysplastic lesion of the keratinocyte $[1,2]$. Over a 10 -year period from 1990 to 1999, AK was diagnosed in more than 47 million visits and was found to occur in $14 \%$ of all patients visiting dermatologists [3]. AK may be treated due to concern for carcinogenesis, patient discomfort, or cosmesis. The most common treatment for AK is cryotherapy [4], likely due to easy access, ease of care for patients, and speed of the procedure. Other management methods include observation, alternative destructive therapies, topical chemotherapies, chemical peels, photodynamic therapy (PDT), and preventative measures such as sunscreen [5]. Given the high prevalence of $\mathrm{AK}$ and the variety and number of clinicians within an organization that encounter AK, CPG can serve as a resource upon which management approaches are unified.
Clinical practice guidelines (CPG) can guide clinicians' diagnostic and therapeutic decisions by succinctly reviewing the literature and proposing evidence-based management recommendations. CPG are defined by the Institute of Medicine as "statements that include recommendations intended to optimize patient care that are informed by a systematic review of evidence and an assessment of the benefits and harms of alternative care options. Rather than dictating a one-size-fits-all approach to patient care, CPG offer an evaluation of the breadth and quality of the relevant scientific literature and an assessment of the likely benefits and harms of a particular treatment" [6]. Thus, a highquality CPG will include an extensive literature review and an evidence rating scale can help readers be aware of the potential limitations of the sources. The quality of CPG can vary for many reasons including the adequacy of the literature search, types of studies incorporated, and bias by the authors 
$[7,8]$. To our knowledge, there is no published assessment of AK CPG quality. We used the AGREE II instrument [9] to assess the strengths and weaknesses of CPG addressing multiple modalities of AK management for patients without immunosuppression.

\section{Methods}

The PRISMA checklist was used as a guide for this investigation. A medical librarian performed a systematic search of the medical literature for CPG with recommendations for $\mathrm{AK}$. An extensive search of Medline/PubMed (1966-March 20, 2014) was conducted for relevant clinical practice guidelines using a combination of the key terms "actinic" and "keratosis" and "guideline" as well as "keratosis, actinic" as a major medical subject heading. Results were limited to practice guideline and guideline publication types. There were no date or language restrictions. In addition the National Guideline Clearinghouse, National Institute for Health and Clinical Excellence, European Academy of Dermatology and Venereology, Guidelines International Network, TRIP Database, Australian Government Clinical Practice Guidelines Portal, Scottish Intercollegiate Guidelines Network, Guidelines and Audit Implementation Network, Professional Organizations and Royal Colleges, Health Service Executive Guidelines and the American Academy Dermatology web sites were searched using key terms "actinic" and "keratosis."

The inclusion criteria were established a priori and (1) included an explicit statement identifying the document as a management guideline, (2) were written by multiple authors including at least one dermatologist, and (3) made recommendations concerning multiple options to manage AK. Exclusion criteria included (1) consensus statements or review articles that did not include identifiable management recommendations, (2) reviews of a published guideline, (3) focus on only preventative, epidemiology, or research methods, (4) focus on a limited or specific patient population, and (5) discussion of one form of management.

Two reviewers (TS and JK) independently examined the retrieved titles and abstracts to assess the articles for inclusion and exclusion criteria. The full text of these articles was retrieved and the same two reviewers (TS and JK) reviewed the selected articles again for eligibility. Disagreement was resolved through discussion by the reviewers (TS and JK). Seven of the guidelines were selected for analysis.

The AGREE II tool was used to describe the quality of the selected CPG. It was developed to evaluate the validity and feasibility of CPG as well as assess sources of bias [9]. The AGREE and AGREE II tools have been validated and widely applied, including CPG of dermatologic conditions $[17,18]$ as well as in other disciplines [19-21]. Four of the authors independently reviewed and scored the CPG. JK and ES are dermatologists, TS was a first-year medical student, and HA is a family practitioner. All four reviewers read the AGREE II users' manual and completed the online orientation and training.

The AGREE II instrument was used to define the assessment variables and rating system and to store data [9]. This instrument provides criteria to appraise the quality of
CPG and consists of 23 items grouped into six domains: (1) scope and purpose, (2) stakeholder involvement, (3) rigor of development, (4) clarity of presentation, (5) applicability, and (6) editorial independence. Each item is rated on a seven-point Likert scale from strongly disagree to strongly agree (1-7, resp.). The results for each domain consist of a domain score, which is the sum of the scores of the individual domain items and standardized by scaling the total as a percentage of the maximum possible score for that domain. The maximum score for each domain was the number of questions multiplied by 7 (strongly agree). The minimum score was the number of questions multiplied by number 1 (strongly disagree):

$$
\begin{aligned}
& \text { Scaled Domain Score } \\
& \begin{aligned}
=\frac{(\text { Sum of Domain scores })-(\text { Minimum possible score })}{(\text { Maximum possible score })-(\text { Minimum possible score })} \\
\quad \times 100 \%
\end{aligned}
\end{aligned}
$$

The minimum standardized score for each domain was, therefore, $0 \%$ and the maximum was $100 \%$. A standardized domain score above $60 \%$ has been suggested as the threshold to indicate sufficient minimum quality to consider practical use of this portion of the guideline [18]. This study was exempted from review by the Penn State Institutional Review Board.

2.1. Statistical Analysis. The scores were compiled and reviewed; upon visual inspection one investigator had scores that appeared to deviate from the other three. The Pearson correlation coefficient is very sensitive to outlying values so Spearman's Rho was used to determine interrater reliability [22]. The degree of agreement was classified as follows: poor (0.00), slight (0.00 and 0.20$)$, fair (0.21 to 0.40$)$, moderate ( 0.41 to 0.60 ), substantial (from 0.61 to 0.80 ), and very good or almost perfect (0.81 to 1.00) [23]. A descriptive statistical analysis of the scores was performed and included the mean and standard deviation. A $p$ value of $<.05$ was considered significant. All analyses were performed using SAS 9.3 (SAS, Cary, NC).

\section{Results}

The search strategy identified 2,307 records and after review ultimately seven records were included in the study (Figure 1). The characteristics of the seven CPG are listed in Table 1. Of the seven CPG, one was from Australia [11], one was from Britain [12], three were from Germany [1315], one was from Italy [16], and one was from the United States [10]. The range for interrater agreement was 0.39-0.86. Analysis showed that one appraiser had only fair agreement with other appraisers $(0.39[p=.38])$. This rater was a firstyear medical student and it was presumed that this reflected clinical experience rather than CPG quality so his scores were removed and subsequent calculations did not include these scores. The correlation coefficients among the three remaining appraisers, which included two dermatologists 


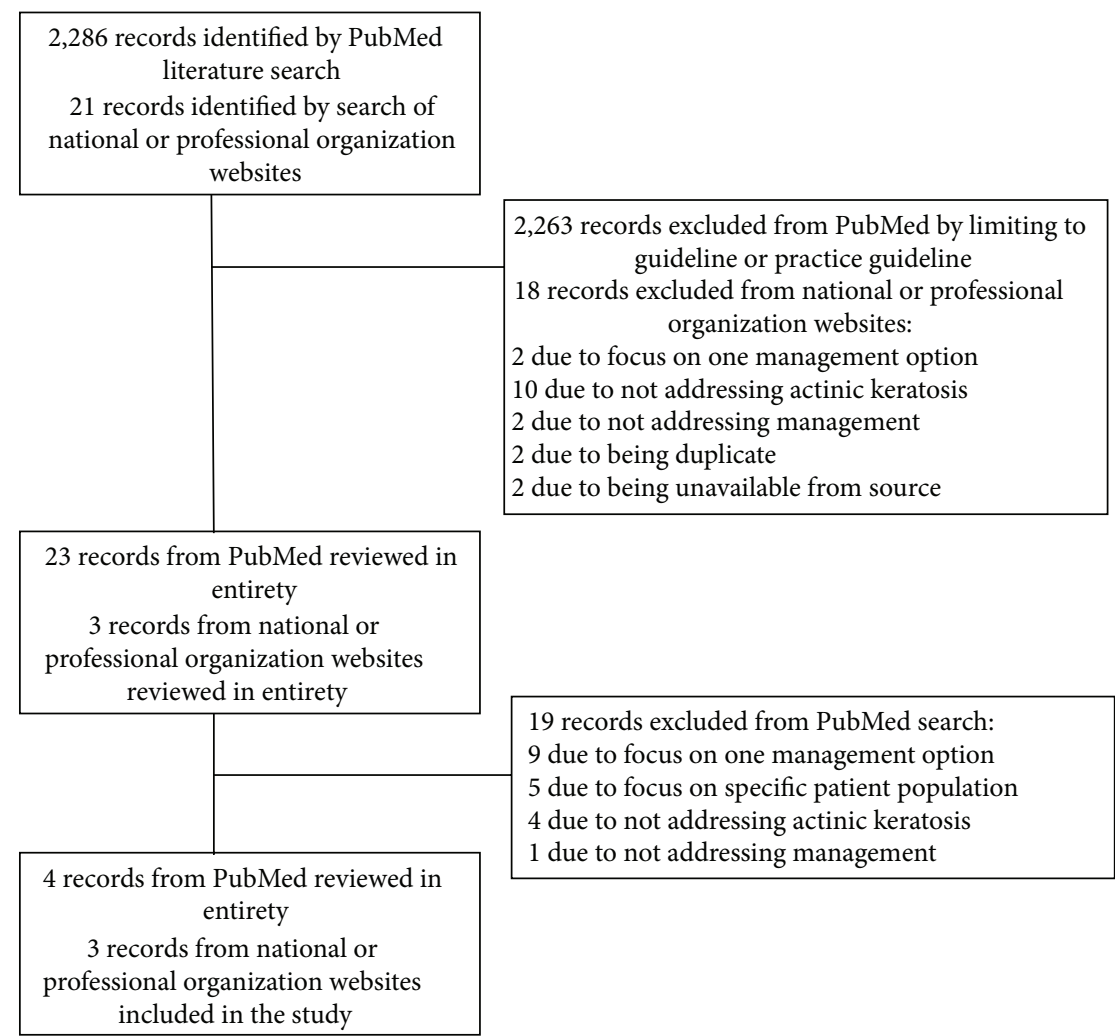

FIgURE 1: Literature search and study selection process.

TABLE 1: Characteristics of the included studies.

\begin{tabular}{lcccc}
\hline Guideline & $\begin{array}{c}\text { Systematic review } \\
\text { performed }\end{array}$ & $\begin{array}{c}\text { Evidence grading } \\
\text { reported }\end{array}$ & $\begin{array}{c}\text { CPG funding } \\
\text { reported }\end{array}$ & $\begin{array}{c}\text { Competing interests } \\
\text { reported }\end{array}$ \\
\hline Drake et al. [10] & NR & No & NR & NR \\
CCA/ACN [11] & Yes & Yes & Yes & Yes \\
de Berker et al. [12] & No & Yes & NR & Yes \\
Stockfleth and Kerl (2006) [13] & NR & Yes & NR & Yes \\
Stockfleth et al. (2008) [14] & NR & No & Yes & Yes \\
Stockfleth et al. (2011) [15] & NR & Yes & NR & Yes \\
Rossi et al. [16] & NR & No & NR & NR \\
\hline
\end{tabular}

CCA/ACN: Cancer Council Australia/Australian Cancer Network. NR = not reported.

and a family practitioner, were moderate or better $(0.54-$ $0.86)$.

The CCA/ACN guideline [11] was the only CPG to perform a systematic review, include evidence-based ratings for recommendations, and report conflicts of interest and funding sources (Table 1). It was also the only CPG to have scores for all domains above $60 \%$ and had the highest score for each of the six domains and overall category (Table 2). The domains with the highest mean scores were domain 1 (scope and purpose) and domain 4 (clarity of presentation) with means of $50.2 \%$ and $51.1 \%$, respectively. The domains with the lowest mean scores were domain 5 (applicability) and domain 6 (editorial independence) with means of $28.6 \%$ and $32.1 \%$, respectively. Domain 6 (editorial independence) also had the lowest individual score of 6.3 and the greatest range in individual scores $(8.3 \%$ to $80.6 \%)$. This may reflect wide variation in disclosure of funding and conflict of interests. While five of the seven CPG included a conflict of interests statement, only two had a disclosure regarding funding sources (Table 1).

Three guidelines were recommended by at least three raters (CCA/CAN [11], de Berker et al. [12], Stockfleth and Kerl 2006 [13]). The CPG by Stockfleth et al. 2008 [14] was recommended by two of the three raters. This second article by Stockfleth et al. 2008 [14] had higher scores in domains 1 (scope and purpose), 2 (stakeholder involvement), 4 (clarity of presentation), and 5 (applicability) but lower scores in domains 3 (rigor of development) and 6 (editorial independence). Domain 3's (rigor of development) score may have been lower due to a lack of a systematic review and 


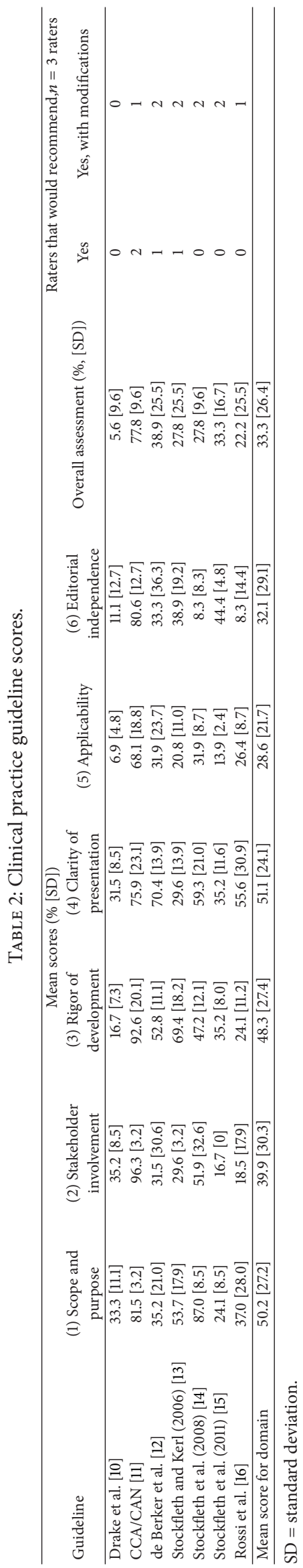


TABLE 3: Samples of recommendations from the three CPG with the highest overall scores.

\begin{tabular}{ll}
\hline Guideline & Samples of CPG recommendations \\
& (i) In many cases solar keratosis [AK] regresses spontaneously and uncommonly; it evolves into squamous cell \\
carcinoma & (ii) The chances that an individual solar keratosis [AK] will develop into SCC are extremely small; however when \\
one encounters SCC, the chance that it has arisen in association with solar keratosis is very high \\
(iii) Thickening and tenderness on lateral palpation are signs that a solar keratosis may have developed into \\
invasive squamous cell carcinoma \\
(i) Studies indicate a high spontaneous regression rate in the order of 15-25\% for AKs over a 1-year period and a \\
low rate of malignant transformation, less than one in 1,000 per annum. \\
(ii) No therapy or emollient is a reasonable option for mild AKs \\
(iii) Sun block applied twice daily for 7 months may protect against development of AKs \\
(iv) 5-Fluorouracil cream used twice daily for 6 weeks is effective for up to 12 months in clearance of the majority \\
of AKs; due to side-effects of soreness, less aggressive regimens are often used, which may be effective but have not \\
been fully evaluated \\
(v) Cryosurgery is effective for up to 75\% of lesions in trials comparing it with photodynamic therapy; it may be \\
particularly superior for thicker lesions but may leave scars \\
(i) Cryotherapy is often used and controlled studies are missing. Complete responses differ from 75\% to 98\%; the \\
recurrence rates of AKs have been estimated from 1.2\% to 12\% within a 1-year follow-up period \\
(ii) The clinical experience in AK patients receiving MAL-PDT shows complete response rate of 70-78\% after a \\
single treatment session and 90\% after two treatment sessions one week apart; negative effects of PDT are local \\
pain, risk of photosensitivity (mainly for ALA), and time delay between application of cream and treatment. \\
Photodynamic therapy in comparison to cryotherapy shows significantly better cosmetic results
\end{tabular}

the methods which were reported as "consultation and discussion of best practice was adopted as a means of formulating a consensus of opinion." This article reported funding by a pharmaceutical company that has AK-related products in its portfolio, which may have negatively impacted the scores in domain 6 (editorial independence). The 2011 update by this group (European Dermatology Forum) was recommended by two of the three raters and compared to the CPG from 2006 and 2008; this document had lower scores than the 2006 and 2008 CPG from this group across all domains except for domain 6 (editorial independence). In contrast to the two prior versions, this CPG had a table at the beginning of the document that listed the COI for each author; however there was no explicit statement of funding.

\section{Discussion}

High-quality CPG can supply clinicians with succinct research findings, specific suggestions for management in particular clinical scenarios, and economic considerations for a specific condition [24]. AK is a common skin lesion and many CPG have been published. Our goal was to review the quality of CPG that are broadly applicable to AK management rather than focus on a specific population or treatment. AKs are treated by providers from multiple disciplines with an array of treatments, so it is valuable and increasingly common for CPG to be written by representatives from the stakeholder groups, including generalists, specialists, and patients $[8,25]$. The CCA/ACN was the only guideline to score above $60 \%$ for the stakeholder involvement domain; it was developed by a multidisciplinary "working party" that included dermatologists, pathologists, plastic surgeons, general practitioners, health economists, epidemiologists, and patients.
The CCA/CAN was also the only CPG that utilized a systematic review and had the highest scores for the rigor of development domain. The results of this review are consistent with previous appraisals of CPG for acne, psoriasis, and pressure ulcers that showed that the rigor of development domain often had lower scores [17, 18]. This domain is important to CPG value since it supports the validity of the recommendations [8]. Other CPG had less rigorous search methods or incomplete descriptions of the methods. CPG help readers to understand the strengths and limitations of the recommendations by including a rating of the supporting evidence. The CCA/ACN, Stockfleth and Kerl 2006 [13], and Stockfleth et al. 2011 [15] CPG used a published evidencebased rating scale, while the de Berker et al. [12] CPG used a rating scale based on evaluator opinion.

CPG rigor is also supported by an evaluation of potential sources of bias, such as conflict of interests (COI). The editorial independence domain includes a rating of COI and funding sources and had the lowest mean score of all the domains. COI reporting by the CPG contributors varied greatly. The Stockfleth et al. 2008 [14] article does address COI and discloses a grant that was received but failed to divulge that the grant came from a pharmaceutical group that produces topical fluorouracil and topical diclofenac products. The CCA/ACN and Stockfleth et al. 2011 [15] CPG clearly listed COI. Other articles (Rossi et al., Drake et al.) [10, 16] lacked a COI statement. The clearly and specifically worded $\mathrm{COI}$ and funding statements offered in the CCA/ACN guideline likely contributed to its having the highest mean score in this domain.

One of the primary purposes of CPG is to make practical, evidence-based recommendations about management of a condition. A summary of the recommendations from the three CPG with the highest scores is presented in Table 3. 
All seven CPG addressed common topical and destructive therapies. In addition, sun avoidance and sun-protection were included in all CPG as a part of $\mathrm{AK}$ prevention and management. Three CPG $[11,12,16]$ summarized evidence that AKs have a low rate of malignant transformation, but only one [12] mentioned observation, emollients, and sunscreen as possible management options [26].

The results of this study should be considered in the context of the limitations, including CPG that were not included and the effects of score variation between raters and within raters. Since the literature search was completed in 2014, this review does not include CPG published more recently which may be valuable $[27,28]$. This review included four reviewers but the scores for one reviewer were not used because they did not correlate well with the others. Also, the CPG were appraised according to the information contained within them and additional supporting documents were not evaluated. It is possible that additional information may have increased scores for some of the CPG but was simply missing from the published version of the guidelines. Also, it is important to consider that high-quality methods or well-written CPG do not necessarily produce valid recommendations.

Effective CPG are evidence-based with recommendations that are concise and organized, so practical application is facilitated [29-31]. The CCA/ACN guideline had the highest scores and included a clear, organized, and concise table at the beginning of the document with helpful evidencelinked recommendations. However, the reviewers found that the recommendations lacked sufficient detail to apply in a clinical setting. For example, the recommendations included "cryotherapy achieves consistently high cure rates for solar keratosis" but lacks details that would facilitate use, such as the duration of the freeze-thaw cycle or number of freezethaw cycles. Also, the CCA/ACN AK recommendations were interspersed among the recommendations for nonmelanoma skin cancer. This made identifying the AK-specific information challenging. Stockfleth et al. [14] included a pictorial algorithm and raters thought this was an effective method to present information, yet scores in other domains lagged behind the CCA/ACN CPG. In closing, CPG are useful tools to guide clinical practice based on existing evidence; however it is important for providers and guideline authors to be aware of the features that contribute to a high-quality, practical, and valuable document.

\section{Disclosure}

This study was exempted from review by the Penn State IRB. This work was presented as a poster at the Society for Investigative Dermatology Annual Meeting, May 7-10, 2015, Atlanta, Georgia.

\section{Disclaimer}

The department specifically disclaims responsibility for any analyses, interpretations, or conclusions. The sponsor was not involved in the design and conduct of the study; collection, management, analysis, and interpretation of data; preparation, review, or approval of the paper; or decision to submit the paper for publication.

\section{Conflict of Interests}

The authors have no conflict of interests related to this work.

\section{Acknowledgments}

The authors would like to thank Amy Knehans, MLS, for expertly performing the literature searches included in this study. This study was supported in part by a career development awarded to Dr. Kirby from the Dermatology Foundation and, in part, under a grant from the Pennsylvania Department of Health using Tobacco CURE Funds.

\section{References}

[1] V. J. Marks, "Actinic keratosis. A premalignant skin lesion," Otolaryngologic Clinics of North America, vol. 26, no. 1, pp. 2335, 1993.

[2] R. A. Schwartz, "The actinic keratosis: a perspective and update," Dermatologic Surgery, vol. 23, no. 11, pp. 1009-1019, 1997.

[3] A. K. Gupta, E. A. Cooper, S. R. Feldman, and A. B. Fleischer Jr., "A survey of office visits for actinic keratosis as reported by NAMCS, 1990-1999. National Ambulatory Medical Care Survey," Cutis, vol. 70, no. 2, supplement, pp. 8-13, 2002.

[4] The Lewin Group, The Burden of Skin Diseases 2004, The Society for Investigative Dermatology and The American Academy of Dermatology Association, Washington, DC, USA, 2005.

[5] A. K. Gupta, M. Paquet, E. Villanueva, and W. Brintnell, "Interventions for actinic keratoses," The Cochrane Database of Systematic Reviews, vol. 12, no. 12, Article ID CD004415, 2012.

[6] IOM (Institute of Medicine), Clinical Practice Guidelines We Can Trust, The National Academics Press, Washington, DC, USA, 2011, http://iom.nationalacademies.org/ /media/Files/ Report\%20Files/2011/Clinical-Practice-Guidelines-We-CanTrust/Clinical\%20Practice\%20Guidelines\%202011\%20Insert .pdf.

[7] P. Alonso-Coello, A. Irfan, I. Solà et al., "The quality of clinical practice guidelines over the last two decades: a systematic review of guideline appraisal studies," Quality and Safety in Health Care, vol. 19, no. 6, article e58, 2010.

[8] A. S. Detsky, "Sources of bias for authors of clinical practice guidelines," Canadian Medical Association Journal, vol. 175, no. 9, pp. 1033-1035, 2006.

[9] M. C. Brouwers, M. E. Kho, G. P. Browman et al., "AGREE II: advancing guideline development, reporting and evaluation in health care," Canadian Medical Association Journal, vol. 182, no. 18, pp. E839-E842, 2010.

[10] L. A. Drake, R. I. Ceilley, R. L. Cornelison et al., "Guidelines of care for actinic keratoses. Committee on Guidelines of Care," Journal of the American Academy of Dermatology, vol. 32, no. 1, pp. 95-98, 1995.

[11] Cancer Council Australia and Australian Cancer Network, "Basal cell carcinoma, squamous cell carcinoma (and related lesions) - a guide to clinical management in Australia," Contract, Cancer Council Australia and Australian Cancer Network, Sydney, Australia, 2014.

[12] D. de Berker, J. M. McGregor, and B. R. Hughes, "Guidelines for the management of actinic keratoses," The British Journal of Dermatology, vol. 156, no. 2, pp. 222-230, 2007. 
[13] E. Stockfleth and H. Kerl, "Guidelines for the management of actinic keratoses," European Journal of Dermatology, vol. 16, no. 6, pp. 599-606, 2006.

[14] E. Stockfleth, C. Ferrandiz, J. J. Grob, I. Leigh, H. Pehamberger, and H. Kerl, "Development of a treatment algorithm for actinic keratoses: a European consensus," European Journal of Dermatology, vol. 18, no. 6, pp. 651-659, 2008.

[15] E. Skockfleth, D. Terhorst, L. Braathen et al., Guidelines for the Management of Actinic Keratoses, European Dermatology Forum, 2010.

[16] R. Rossi, P. G. Calzavara-Pinton, A. Giannetti et al., "Italian guidelines and therapeutic algorithm for actinic keratoses," Giornale Italiano di Dermatologia e Venereologia, vol. 144, no. 6, pp. 713-723, 2009.

[17] E. R. M. de Haas, H. C. de Vijlder, S. W. van Reesema, J. J. E. van Everdingen, and H. A. M. Neumann, "Quality of clinical practice guidelines in dermatological oncology," Journal of the European Academy of Dermatology and Venereology, vol. 21, no. 9, pp. 1193-1198, 2007.

[18] G. Sanclemente, J.-L. Acosta, M.-E. Tamayo, X. Bonfill, and P. Alonso-Coello, "Clinical practice guidelines for treatment of acne vulgaris: a critical appraisal using the AGREE II instrument," Archives of Dermatological Research, vol. 306, no. 3, pp. 269-277, 2014.

[19] A. Lo Vecchio, A. Giannattasio, C. Duggan et al., "Evaluation of the quality of guidelines for acute gastroenteritis in children with the AGREE instrument," Journal of Pediatric Gastroenterology and Nutrition, vol. 52, no. 2, pp. 183-189, 2011.

[20] A. Hurdowar, I. D. Graham, M. Bayley, M. Harrison, S. WoodDauphinee, and S. Bhogal, "Quality of stroke rehabilitation clinical practice guidelines," Journal of Evaluation in Clinical Practice, vol. 13, no. 4, pp. 657-664, 2007.

[21] T. Lytras, S. Bonovas, C. Chronis et al., "Occupational asthma guidelines: a systematic quality appraisal using the AGREE II instrument," Occupational and Environmental Medicine, vol. 71, no. 2, pp. 81-86, 2014.

[22] M. Pagano and K. Gauvreau, Eds., Principles of Biostatistics, Brooks/Cole, Belmont, Calif, USA, 2nd edition, 2000.

[23] M. S. Kramer and A. R. Feinstein, "Clinical biostatistics. LIV. The biostatistics of concordance," Clinical Pharmacology and Therapeutics, vol. 29, no. 1, pp. 111-123, 1981.

[24] M. J. Field and K. Lohr, Eds., Clinical Practice Guidelines: Directions for a New Program, Institute of Medicine, Washington, DC, USA, 1990.

[25] J. N. Lavis, "How can we support the use of systematic reviews in policymaking?" PLoS Medicine, vol. 6, no. 11, Article ID e1000141, 2009.

[26] R. N. Werner, A. Sammain, R. Erdmann, V. Hartmann, E. Stockfleth, and A. Nast, "The natural history of actinic keratosis: a systematic review," The British Journal of Dermatology, vol. 169, no. 3, pp. 502-518, 2013.

[27] B. Dréno, J. M. Amici, N. Basset-Seguin, B. Cribier, J. P. Claudel, and M. A. Richard, "Management of actinic keratosis: a practical report and treatment algorithm from AKTeam expert clinicians," Journal of the European Academy of Dermatology and Venereology, vol. 28, no. 9, pp. 1141-1149, 2014.

[28] R. N. Werner, E. Stockfleth, S. M. Connolly et al., Evidence and Consensus Based (S3) Guidelines for the Treatment of Actinic Keratosis International League of Dermatological Societies (ILDS) in Cooperation with the European Dermatology Forum (EDF), 2014, http://www.euroderm.org/edf/index.php/edfguidelines/category/5-guidelines-miscellaneous.
[29] E. C. Haagen, W. L. D. M. Nelen, R. P. M. G. Hermens, D. D. M. Braat, R. P. T. M. Grol, and J. A. M. Kremer, "Barriers to physician adherence to a subfertility guideline," Human Reproduction, vol. 20, no. 12, pp. 3301-3306, 2005.

[30] M. D. Cabana, C. S. Rand, N. R. Powe et al., "Why don't physicians follow clinical practice guidelines? A framework for improvement," The Journal of the American Medical Association, vol. 282, no. 15, pp. 1458-1465, 1999.

[31] A. L. Francke, M. C. Smit, A. J. E. De Veer, and P. Mistiaen, "Factors influencing the implementation of clinical guidelines for health care professionals: a systematic meta-review," $B M C$ Medical Informatics and Decision Making, vol. 8, article 38, 2008. 


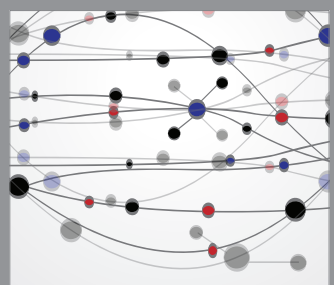

The Scientific World Journal
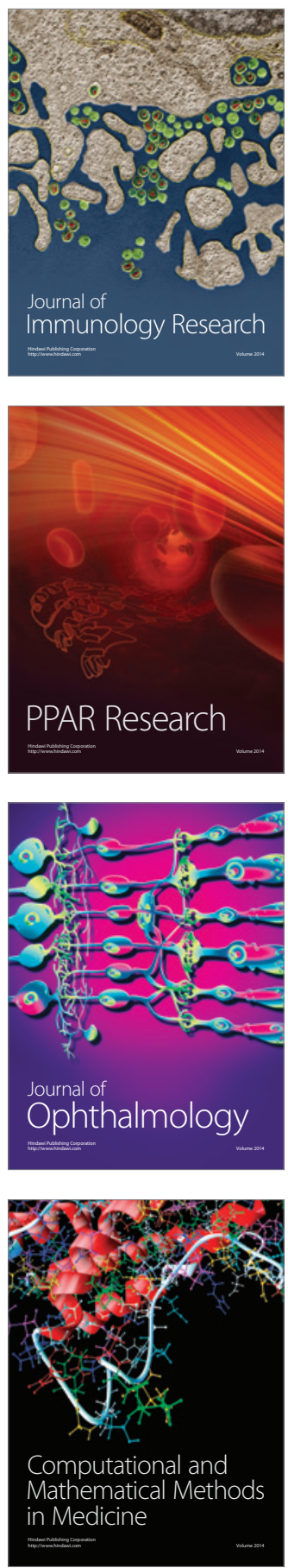

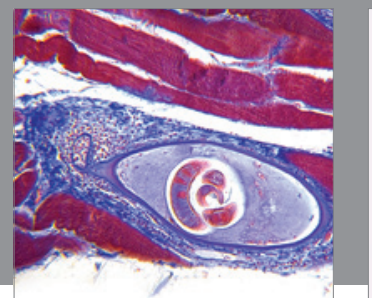

Gastroenterology

Research and Practice
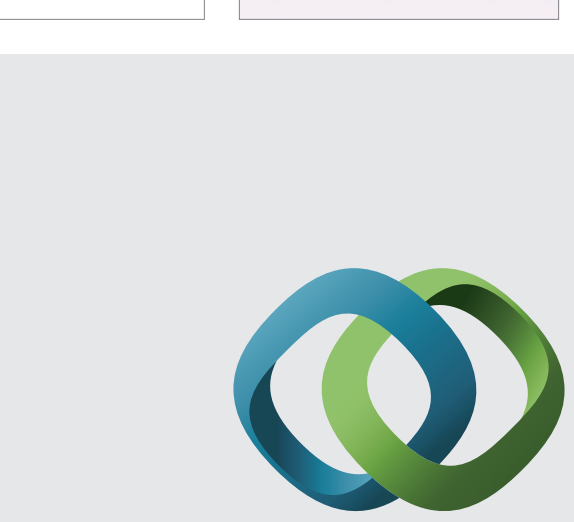

\section{Hindawi}

Submit your manuscripts at

http://www.hindawi.com
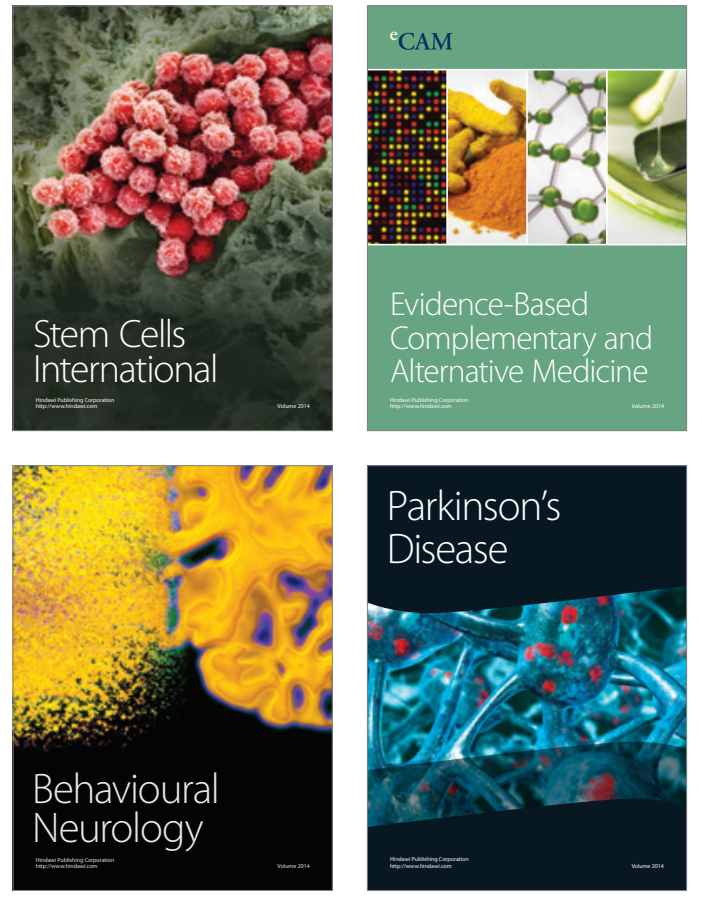
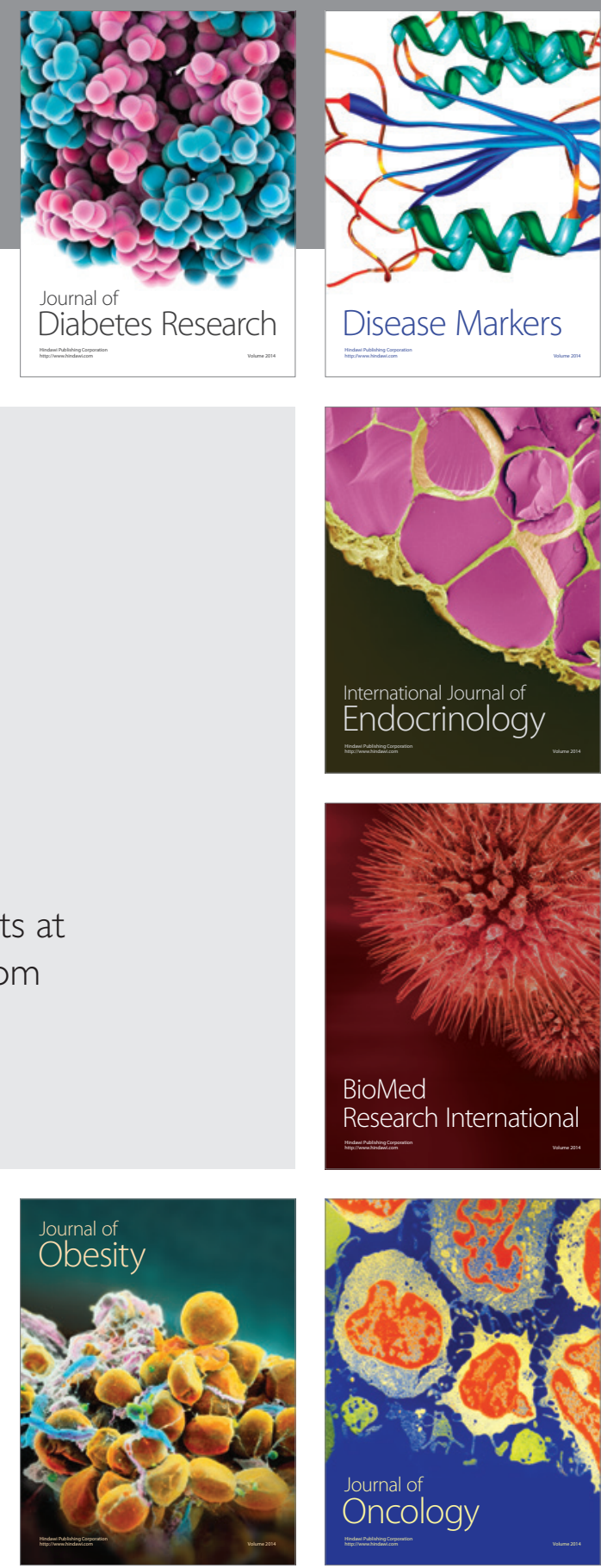

Disease Markers
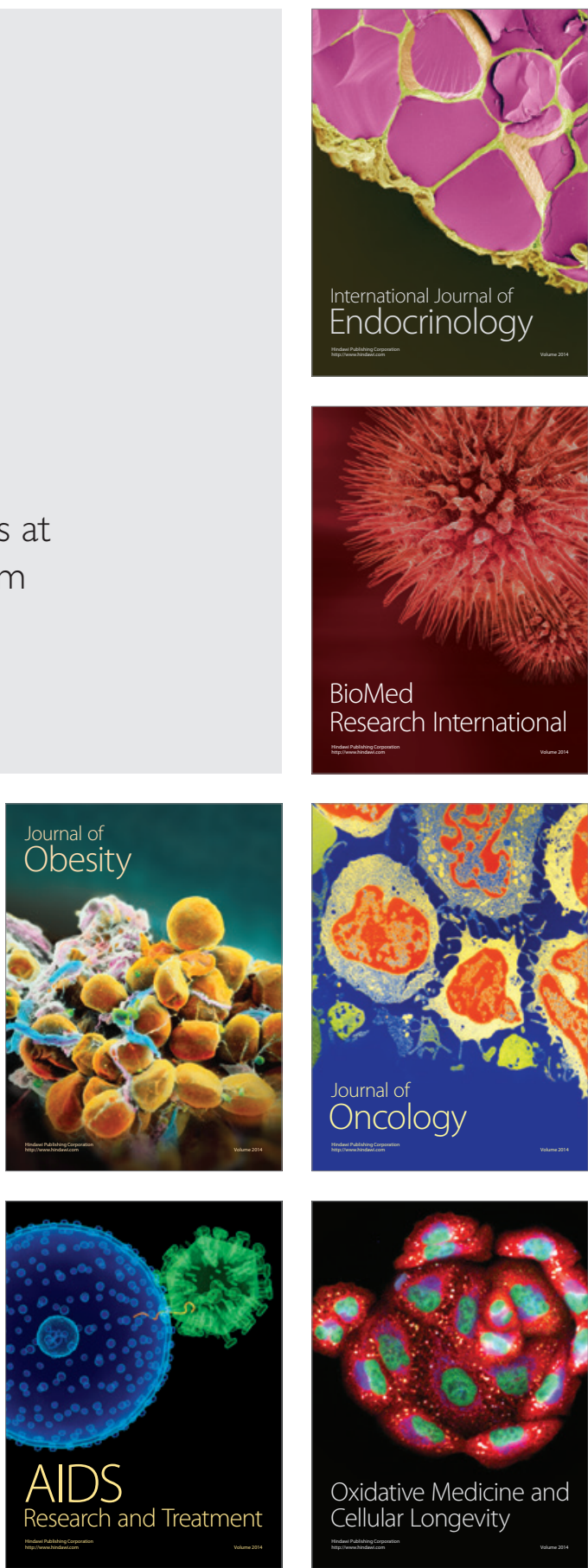